\title{
AN ACCURATE METHOD FOR EXTRACTING THE CRITICAL FIELD IN SHORT CHANNEL NMOS DEVICES
}

\author{
Y. AMHOUCHE, A. EL ABBASSI, K. RAÏS* and R. RMAILY \\ Laboratoire de Caractérisation des Composants à Semi-conducteurs, \\ Université Chouaib Doukkali. B.P. 20, EL Jadida-Maroc
}

(Received 1 April 2001; In final form 15 May 2001)

\begin{abstract}
In this letter, an accurate method for extracting the critical field $E c$ in short channel MOSFET's is presented. The principle of this method is based on the comparison between two models which give drain saturation voltage evolution against gate voltage $V d_{\text {sat }}(V g)$ continuously. The results obtained by this technique have shown better agreement with measurements data and have allow in the same time to determine the validity domain of Sodini's law [1].
\end{abstract}

Keywords: Drain saturation voltage; Critical field; Substrate current; Effective mobility; Saturation velocity

\section{INTRODUCTION}

The effect of velocity saturation on the electric characteristics in short channel MOSFET's has been the subject of several recent studies $[2,3]$. However, most of the existing methods of $E c$ and $v_{\text {sat }}$ extraction, $v_{\text {sat }}$ being saturation velocity, doesn't allows to relate clearly drain saturation voltage to velocity saturation. On the other hand, the application of thornber's equation $\left(v_{\mathrm{sat}}=\mu_{\mathrm{eff}} \cdot E c\right)$ [4], where $\mu_{\mathrm{eff}}$ is the effective mobility, suggest a precise determination of $\mu_{\mathrm{eff}}$ and $E c$ values.

In a previous paper [5], the present authors proposed a new model to characterise drain saturation voltage in short channel MOSFET's

*Corresponding author. 
based on measurements of the partial derivative of impact ionisation rate which has allowed to find $V d_{\text {sat }}(V g)$ characteristics continuously. According to this method and the analytical model of drain saturation voltage given by Sodini [1], a novel technique for determination of the critical field is presented.

\section{EXTRACTION METHOD FORMULATION}

Drain saturation voltage is given by the following analytical expression:

$$
V d_{\mathrm{sat}}(V g)=\frac{E c \cdot L \cdot(V g-V t)}{E c \cdot L+V g-V t}
$$

where $V t, E c, L$ are threshold voltage, the critical field and the channel length respectively, we note that $V t$ is determined using the method described in [6]. The second model which give $V d_{\text {sat }}(V g)$ continuously can be expressed such as [5]:

$$
V d_{\text {sat }}(V g)=-\int_{V t}^{V g} \frac{\partial\left(\frac{I s u b}{I d}\right) / \partial V g}{\partial\left(\frac{I s u b}{I d}\right) / \partial V d}
$$

where $I d$ is the drain current and $I s u b$ represent the substrate current. $V d$ being the drain voltage. From Eq. (1) we define the function $F$ as follows:

$$
F(V g)=\frac{1}{\sqrt{\frac{\partial V d_{\text {sat }}}{\partial V g}}}(V g)=1+\frac{1}{E c L}(V g-V t)
$$

On the other hand, using Eq. (2), $V d_{\text {sat }}$ derivative on $V g$ can be obtained simply such as:

$$
\frac{\partial V d_{\mathrm{sat}}}{\partial V g}=-\frac{\partial\left(\frac{I s u b}{I d}\right) / \partial V g}{\partial\left(\frac{I s u b}{I d}\right) / \partial V d}
$$


The plot of $F(V g)$ against ( $V g-V t)$, using Eq. (4) for $F(V g)$ values determination, leads directly to the critical field value and provide a simple manner of testing the adequacy of $V d_{\text {sat }}$ law of Eq. (1) and it's validity domain.

\section{RESULTS AND DISCUSSION}

The previous analysis is applied at room temperature to $\mathrm{N}$ Channel MOSFET devices, with channel width $W$ is $50 \mu \mathrm{m}$. The channel lengths are in the range $0.3-1.5 \mu \mathrm{m}$, the channel doping $N a$ ranging between $10^{15}$ and $10^{16} / \mathrm{cm}^{3}$, the oxide thickness $t_{\mathrm{ox}}$ is $15 \mathrm{~nm}$.

The Figure 1 show the application of the partial derivative method [5] in comparison with chan et al. [7]. As seen in this figure, the partial derivative method can be used successfully to found $V d_{\text {sat }}(V g)$ characteristics continuously for short channel lengths. In Figure 2 is displaying typical $F(V g)$ characteristics representative for

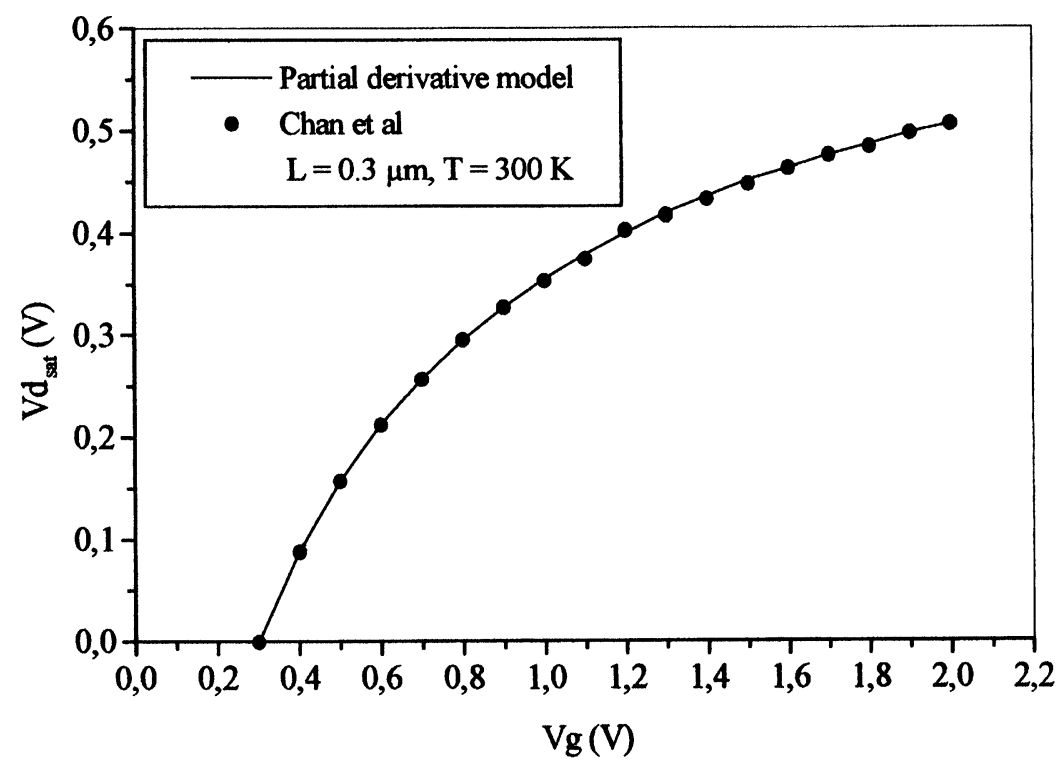

FIGURE $1 V d_{\text {sat }}(V g)$ characteristics for $0.3 \mu \mathrm{m}$ channel length at $T=300 \mathrm{~K}$. 


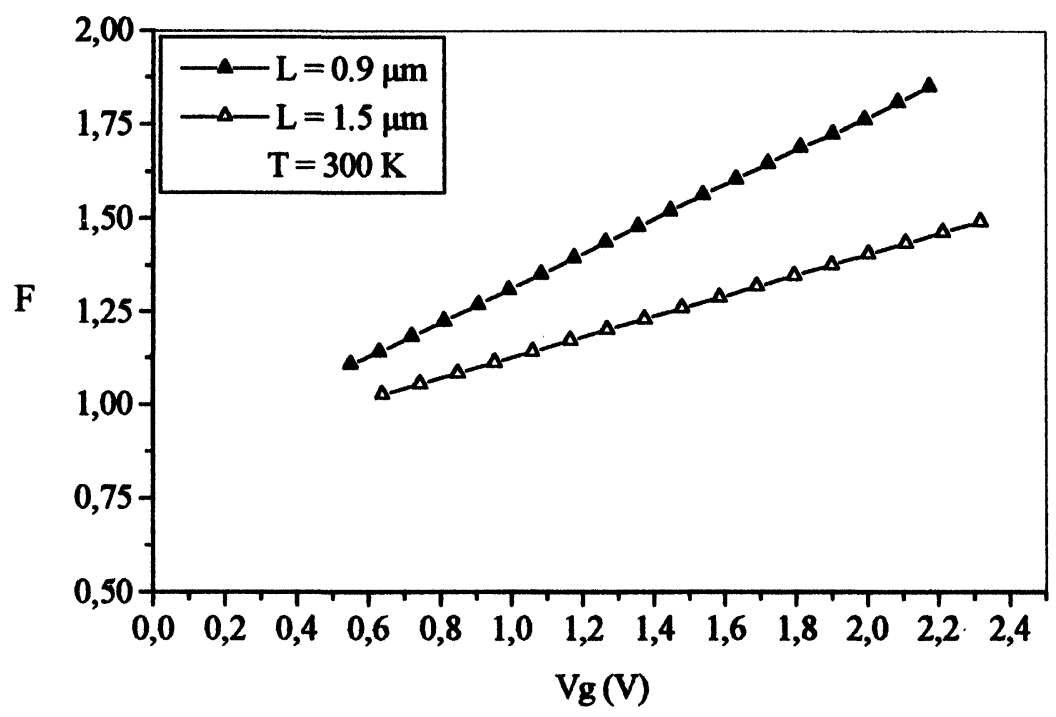

FIGURE 2 Evolution of the function $F$ against gate voltage $V g$ for $L=0.9$ and $1.5 \mu \mathrm{m}$.

two different channel lengths, $L=0.9$ and $1.5 \mu \mathrm{m}$. As shown in this figure, these plot exhibit, in agreement with our analysis, an excellent linear dependence with gate voltage. It should be mentioned that this test of linearity, which indirectly confirms the validity of the method has been successfully completed on other channel lengths but not smaller than $0.6 \mu \mathrm{m}$ because the domain validity of Eq. (1) is found to be limited in the range $0.6-1.5 \mu \mathrm{m}$. The extracted value of $E c$ is $2,45 \cdot 10^{6} \mathrm{~V} / \mathrm{m}$ for $L=0.9 \mu \mathrm{m}$ and $2,5 \cdot 10^{6} \mathrm{~V} / \mathrm{m}$ for $L=1.5 \mu \mathrm{m}$ which is in very good agreement with literature results which indicate that $E c$ is almost constant with channel length. For $L=0.3 \mu \mathrm{m}$ (Fig. 3), the plot linearity is not clear, this can be explained by the fact that our approach is based on Eq. (1) which itself based on a simplified considerations. On the other hand, the partial derivative method allows a good description of $V d_{\text {sat }}(V g)$ evolution even for very short channels. Therefore, the extracted value of $E c$ in this case is not done because Eq. (1) doesn't take into account rigorously of the non linear dependence of drift velocity on the electric field and can not be used reliably to describe 


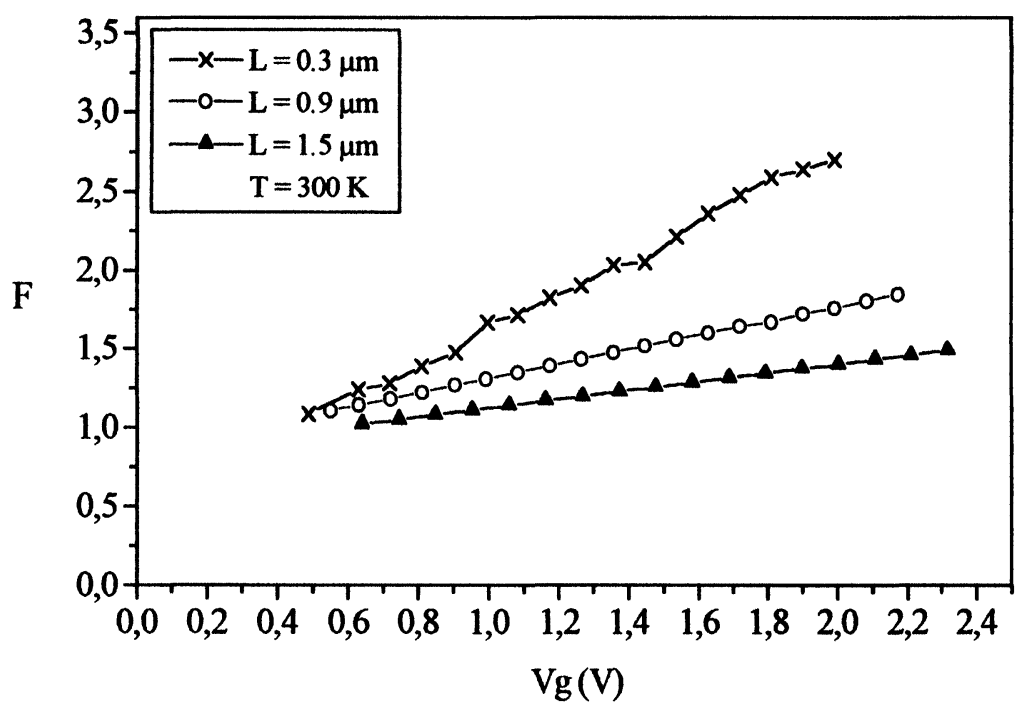

FIGURE $3 F(V g)$ characteristics for $L=0.3,0.9$ and $1.5 \mu \mathrm{m}$.

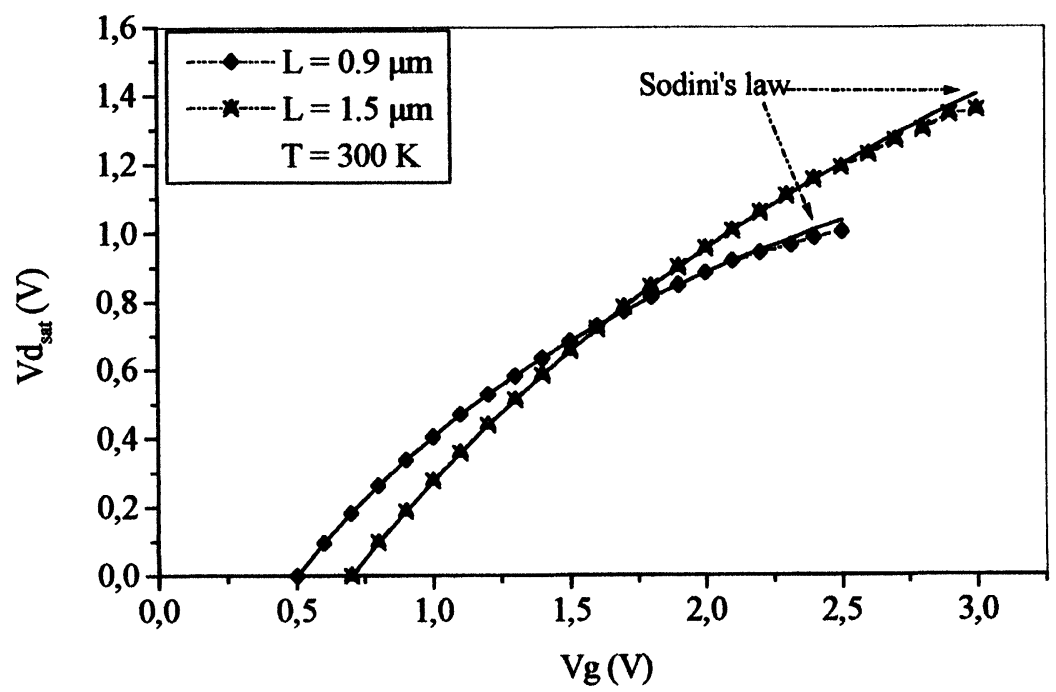

FIGURE 4 Measured and theoretical $V d_{\text {sat }}$ against $V g$ for MOSFET's with $L=0.9$ and $1.5 \mu \mathrm{m}$ channel lengths. 
$V d_{\text {sat }}(V g)$ evolution for very short channel lengths. In Figure 4 is shown the application of the partial derivative model and Chan et al. method for $V d_{\text {sat }}$ determination in comparison with Sodini's law after $E c$ extraction. The superposition of data justify in the same time the $E c$ extracted values and the method extraction validity in the considered range of lengths.

\section{CONCLUSION}

In this work an accurate method for determination of the critical field in short channel MOSFET's has been presented. It has been demonstrated that the application of Sodini's law for $V d_{\text {sat }}$ determination must be preceded by a precise evaluation of $E c$. On the other hand this method can be advantageously used to provide a simple manner for verification of Eq. (1) validity domain, therefore allowing a novel way for extracting saturation velocity.

\section{References}

[1] C. Sodini, P. K. Ko and J. Moll (1984) IEEE Trans. Electron. Dev ED31, 1386-1393.

[2] K. Rais, G. Ghibaudo, F. Balestra and M. Dutoit (1994) Wolte.

[3] S. Saha, C. S. Yeh and B. Gadepally (1993) Sol. State Electron 36, 1429-1432.

[4] K. K. Thornber (1980) J. App. Phys 51, 2127.

[5] Y. Amhouche, A. El Abbassi, E. Bendada, K. Rais and R. Rmaily (2001) Active and Passive Electronic Components (In press).

[6] G. Ghibaudo and F. Balestra (1989) Solid State Electron 32, 221.

[7] T. Y. Chan, P. K. Ko and C. Hu (1984) IEEE Trans. Electron. Dev 5, 505-507. 

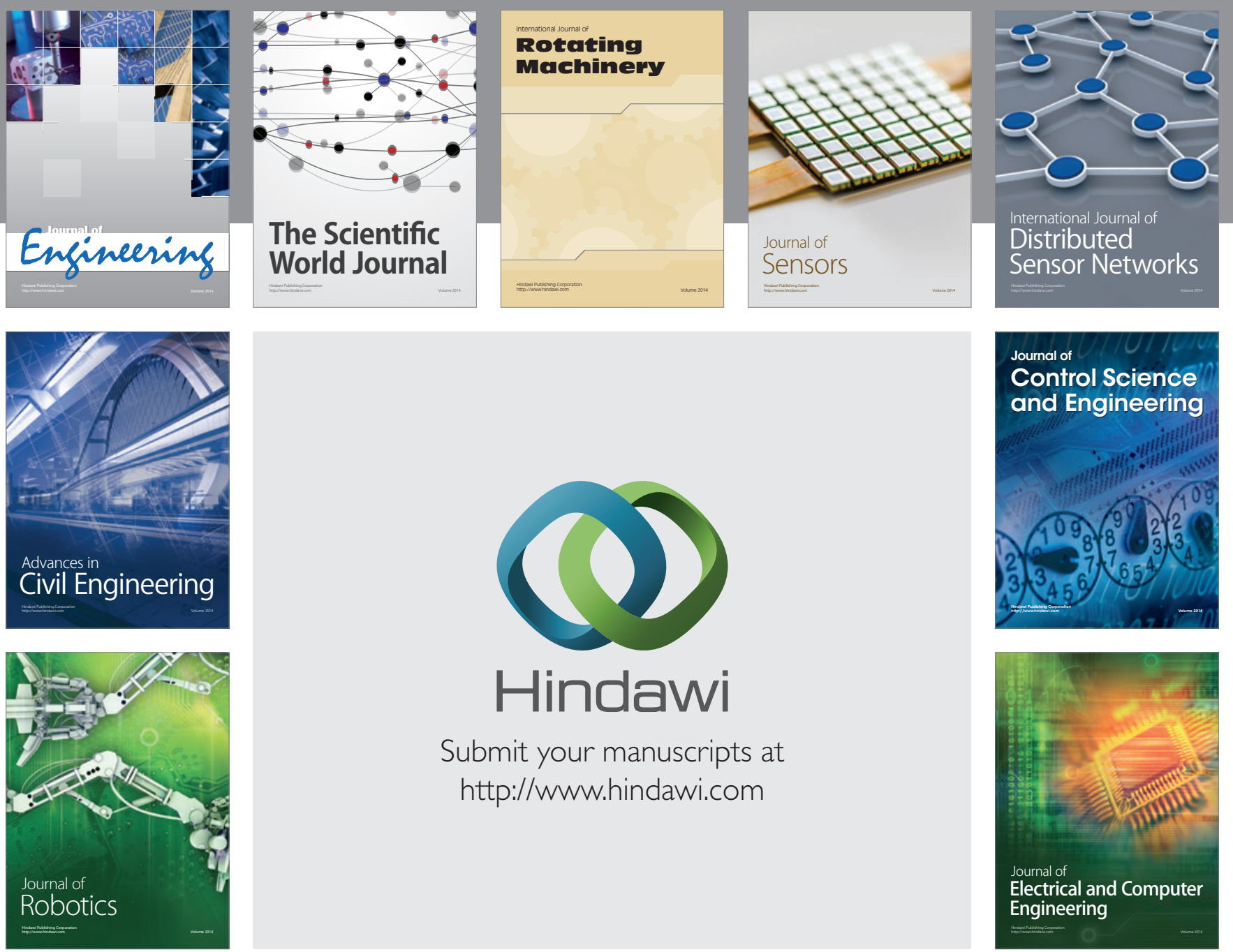

Submit your manuscripts at

http://www.hindawi.com
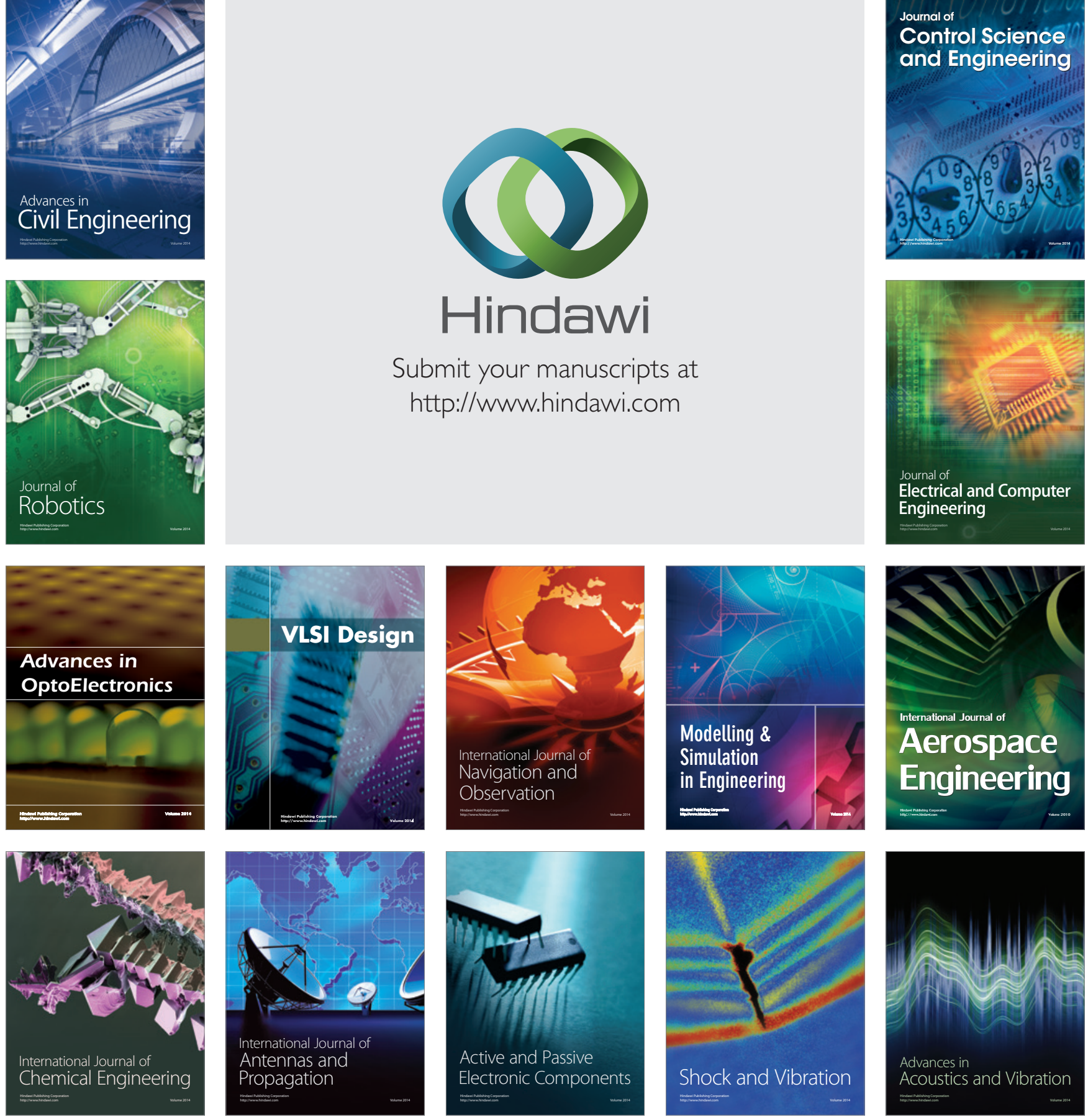